\title{
Magnetic Properties and Structure of the Ni-Co-Mn-In Alloys with the Boron Addition
}

\author{
K. Prusik ${ }^{a, *}$, E. Matyja ${ }^{a}$, M. Kubisztal $^{a}$, M. ZubKo $^{a}$ And R. SwadźBA ${ }^{b}$ \\ ${ }^{a}$ University of Silesia, Institute of Material Science, Bankowa 12, 40-007 Katowice, Poland \\ ${ }^{b}$ Institute for Ferrous Metallurgy, J. Sowińskiego 5, 44-101 Gliwice, Poland
}

\begin{abstract}
Series of $\mathrm{Ni}_{45.5-x} \mathrm{Co}_{4.5} \mathrm{Mn}_{36.6} \operatorname{In}_{13.4} \mathrm{~B}_{x}$ (at.\%, $x=0,0.05,0.1,0.5,1.0$ ) polycrystalline magnetic shape memory alloys were examined in terms of the magnetic properties, structure and transition temperatures. Depending on the boron concentration single or two phase alloys microstructures were observed. Additionally, the martensitic transformation temperatures decreases with the boron addition. Magnetic-field induced transformation occurs for the alloys with the boron addition up to 0.1 at.\%. For alloys with 0.5 and 1.0 at.\% of B transformation is hindered.
\end{abstract}

DOI: 10.12693/APhysPolA.131.1240

PACS/topics: 81.30.Kf, 87.64.Ee, 75.50.Ee, 75.50.Gg, 71.20.Be

\section{Introduction}

Magnetic shape memory alloys (MSMAs) can play a very important role as active materials in many applications. In this alloys shape recovery can be obtained not only by temperature or stress but also by the external magnetic field. In conventional ferromagnetic shape memory (FSMAs) alloys shape change due to the external magnetic field (large magnetostriction) is connected with the magnetic martensite variant reorientation mechanism $[1,2]$. During cooling they undergo a martensitic transformation from the high temperature and highly symmetrical ferromagnetic parent $(\mathrm{P})$ phase to the low temperature less-symmetrical and nonmagnetic (or weak magnetic) martensite (M) phase. Until now magneticfield induced strain of about $10 \%$ was observed in $\mathrm{Ni}-$ $\mathrm{Mn}-\mathrm{Ga}$ single crystals [2]. Apart from the conventional FSMAs there are second group of alloys, called metamagnetic shape memory alloys (MMSMAs), in which shape change may be driven also by external magnetic field. In this alloys, unlike in the conventional FSMAs, shape recovery can be obtained by the magnetic field induced reverse transformation. This metamagnetic transformation occurs from martensite to the ferromagnetic parent phase under external magnetic field $[2,3]$. Until now several alloy systems have been developed as MMSMAs e.g. $\mathrm{Ni}-\mathrm{Mn}-\mathrm{X}$ (In, Sn, Sb) [4]. In $\mathrm{Ni}_{45} \mathrm{Co}_{5} \mathrm{Mn}_{36.7} \mathrm{In}_{13.3}$ single crystal large magnetic-field induced-stress over $100 \mathrm{MPa}$ can be generated [5]. Metamagnetic shape memory effect (MMSME) can be also observed in the polycrystalline materials with preferred grains orientation (textured). There are several methods for introducing texture e.g. by plastic deformation [6], extrusion [7] etc. Unfortunately, monocrystalline as well as polycrystalline MSMAs are very brittle. It is known that a small amount of boron in e.g. Ti-alloys [8], Ni-Mn-Ga [9] and steel [10]

*corresponding author; e-mail: krystian.prusik@us.edu.pl improves their mechanical properties but can also affect the structure and magnetic properties of the alloys.

In this paper the effect of the boron addition on the structure and magnetic properties of the polycrystalline $\mathrm{Ni}_{45.5-x} \mathrm{Co}_{4.5} \mathrm{Mn}_{36.6} \operatorname{In}_{13.4} \mathrm{~B}_{x}$ [at.\%] $x=0,0.05,0.1,0.5$, 1.0 alloys was studied.

\section{Experimental procedure}

Series of $\mathrm{Ni}_{45.5-x} \mathrm{Co}_{4.5} \mathrm{Mn}_{36.6} \mathrm{In}_{13.4} \mathrm{~B}_{x}$ [at.\%] $x=0$, $0.05,0.1,0.5,1.0$ polycrystalline MSMAs (denoted respectively as B000, B005, B010, B050, B100) were prepared by the induction melting. Ingots were homogenized at 1173 for $24 \mathrm{~h}$ in vacuum quartz capsules and subsequently quenched into icy water. The structure was examined by JEOL JEM3010 transmission electron microscope (TEM). The microstructure of the studied alloys was observed by the JEOL JSM-6480 scanning electron microscope (SEM) and FEI TITAN 80-300 S/TEM. Magnetic properties were measured by PPMS7 vibrating sample magnetometer (VSM).

\section{Results and discussion}

\subsection{Structure of the alloys}

Figure 1 shows microstructures of the B000-B100 alloys. For all studied alloys, microstructure of the matrix was characterized by very coarse grains from $200 \mu \mathrm{m}$ to even $\mathrm{mm}$ in size. Depending on the boron addition two type of the microstructures were observed: single (B000) or two phase (B005-B100). In Fig. 1b (B005 alloy) inside the matrix the martensite regions appeared (darker plates). For B010, B050 and B100 the precipitates of the second phase were observed.

Therefore the nucleation of the second phase is favored by the boron addition. The mean size of the precipitated grains is around $1-2 \mu \mathrm{m}$. One can see that the volume fraction of precipitates increases with the boron addition. Chemical analyses done by EDS and WDS revealed that precipitates are richer in $\mathrm{Co}$ and poorer in $\mathrm{Ni}$ 


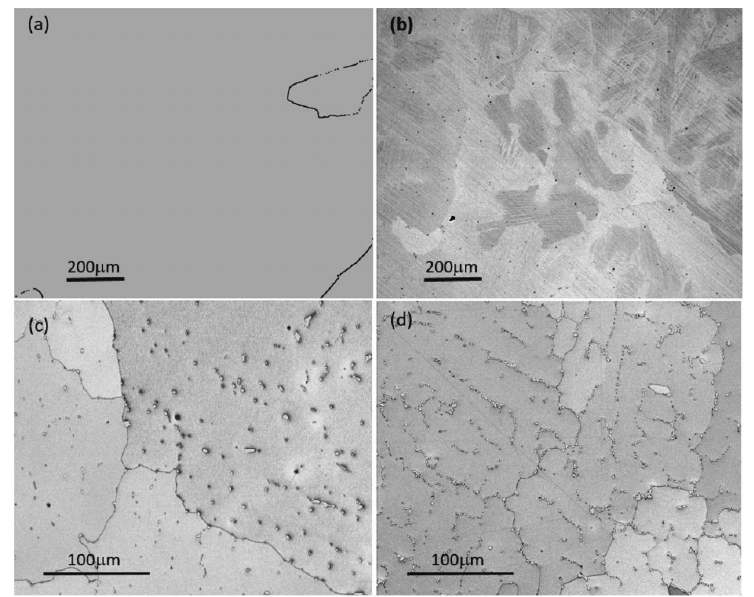

Fig. 1. Microstructure of B000 (a), B005 (b), B050 (c), B100 (d).

than the matrix. The concentration of elements in the precipitates do not changes much with the boron addition. The metallic elements to the boron contents ratio is about $4: 1$. Structure of the matrix and precipitates of the $\mathrm{Ni}_{45.5-x} \mathrm{Co}_{4.5} \mathrm{Mn}_{36.6} \operatorname{In}_{13.4} \mathrm{~B}_{x}$ [at.\%] alloys were studied by TEM. In Fig. 2a bright field TEM image of the precipitate within the matrix of the B050 alloy is depicted. One can see the $\{111\}$ type superlattice reflections in the selected area diffraction pattern (SAED) (Fig. 2c) of the matrix. That proves the $L 2_{1}$ ordering. The space group of the matrix is $F m 3 m$ and the lattice parameter $a_{0}=5.98 \AA$ [11]. The structure of the precipitates of B010-B100 alloys were determined as $\mathrm{M}_{23} \mathrm{~B}_{6}$. Additionally, in the B005 specimen the martensite plates were observed.

The martensite structure has been determined as monoclinic seven-layered $14 \mathrm{M}$ structure (denoted also as $7 \mathrm{M}$ ) with lattice parameter $a_{0}=4.23 \AA, b_{0}=5.62 \AA$, $c_{0}=21 \AA, \alpha=90^{\circ}, \beta=89.9^{\circ}$.

\subsection{Magnetic properties}

Magnetic field induced transformation is very convenient method by which high values of the output stresses can be obtained. For the MMSMAs the magnitude of the output stress is much higher than that observed for the "classic" ferromagnetic shape memory alloys (e.g. Ni$\mathrm{Mn}-\mathrm{Ga}$ ) for which the martensitic variant rearrangement occurs [5]. In MMSMAs, application of the magnetic field change $(\Delta B)$ induces reverse martensitic transformation. Change of the transformation temperature $(\Delta T)$ can be approximately given by the Clausius-Clapeyron relation in the magnetic phase diagram $[5,12]$ :

$$
\frac{\mathrm{d} B}{\mathrm{~d} T}=\frac{\Delta S}{\Delta M} \text { and } \Delta T \approx\left(\frac{\Delta M}{\Delta S}\right) \Delta B,
$$

where $T$ is the temperature (absolute), $B$ is magnetic induction magnitude and $\Delta M$ and $\Delta S$ are the differences in magnetization and entropy between martensite and parent phase, respectively.
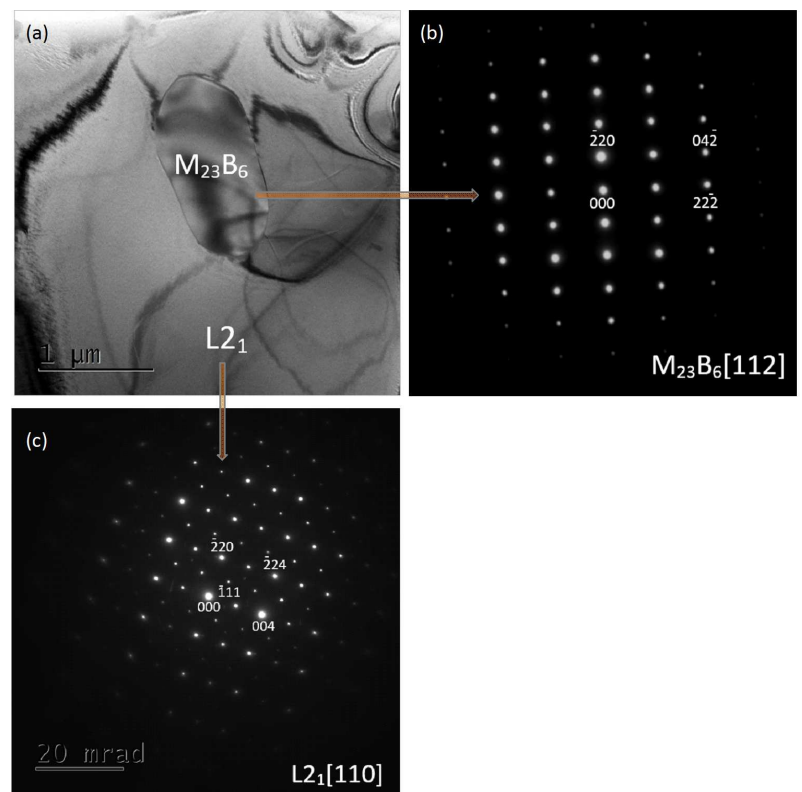

Fig. 2. Bright field TEM image of the B050 alloy (a) and selected area diffraction patterns taken from the precipitate (b) and matrix (c).

In the studied MMSMAs the boron additions strongly influence also the magnetic properties. Figure 3 shows thermomagnetization curves (i.e. the effect of applied magnetic field on the magnetization response as a function of temperature) for B000 (Fig. 3a), B005 (Fig. 3b), B010 (Fig. 3c), and B100 (Fig. 3d) alloys measured under several magnetic fields $(0.5,20,50$, and $70 \mathrm{kOe})$. For B000 sample under magnetic field of 500 Oe, the austenite to martensite transformation starts at $270 \mathrm{~K}\left(T_{M s}\right)$ and finishes at $262 \mathrm{~K}\left(T_{M f}\right)$ upon cooling. The reverse martensite transformation starts at $278 \mathrm{~K}\left(T_{A s}\right)$ and finishes at around $287 \mathrm{~K}\left(T_{A f}\right)$ upon heating. One can see that up to the $20 \mathrm{kOe}$ transformation is fully reversible with a relatively small thermal hysteresis (about $15 \mathrm{~K}$ at $500 \mathrm{Oe})$. As the applied magnetic field increases from 500 Oe to $70 \mathrm{kOe}$, the transformation temperatures decreases; e.g., $M_{s}$ decreases from $270 \mathrm{~K}$ to $147 \mathrm{~K}$. The decrease of the martensite characteristic temperatures with the magnetic field is mainly due to the fact that the applied magnetic field favors the phase with the higher saturation magnetization which for this alloy is austenite. For the B005 specimen so called the abnormal behavior was observed. The temperature changes due to the applied magnetic field $(\Delta T)$ was far less than those observed for B000 (Fig. 3b). For example $\Delta T_{M s}$ (from $0.5 \mathrm{kOe}$ to $70 \mathrm{kOe}$ ) observed for $\mathrm{B} 000$ is about $120 \mathrm{~K}$ whereas for B005 - only $25 \mathrm{~K}$. It is probably because not whole matrix volume but only some part of it transforms to the austenite (martensite) under applied magnetic field upon heating/cooling.

Additionally, for B005 transformation temperature hysteresis is very wide. The $\Delta\left(T_{M s}-T_{M f}\right)$ is over $100 \mathrm{~K}$ whereas for $\mathrm{B} 000$ is about $20 \mathrm{~K}$. For the specimen with 
0.1 at.\% of $\mathrm{B}$ addition the thermomagnetizations curves look similar to the B000. In comparison to the B000, only a slight decrease of the transformation temperatures vs. applied magnetic field was observed. On the other hand, when the content of boron is above the certain value (about 0.5 at. \%B) the thermally activated martensite transformation is inhibited. For the specimens B050 and B100 no transformation induced by magnetic field in the thermomagnetization curves was observed. The transformation is hindered due to the presence of Corich precipitates. So that electron to atom ratio (e/a) of the matrix decreases and as a result the transformation temperatures decreases as well. Figure 4 shows the magnetization curves at $220-360 \mathrm{~K}$ temperature range for B000, B005, B010, and B100 specimens.

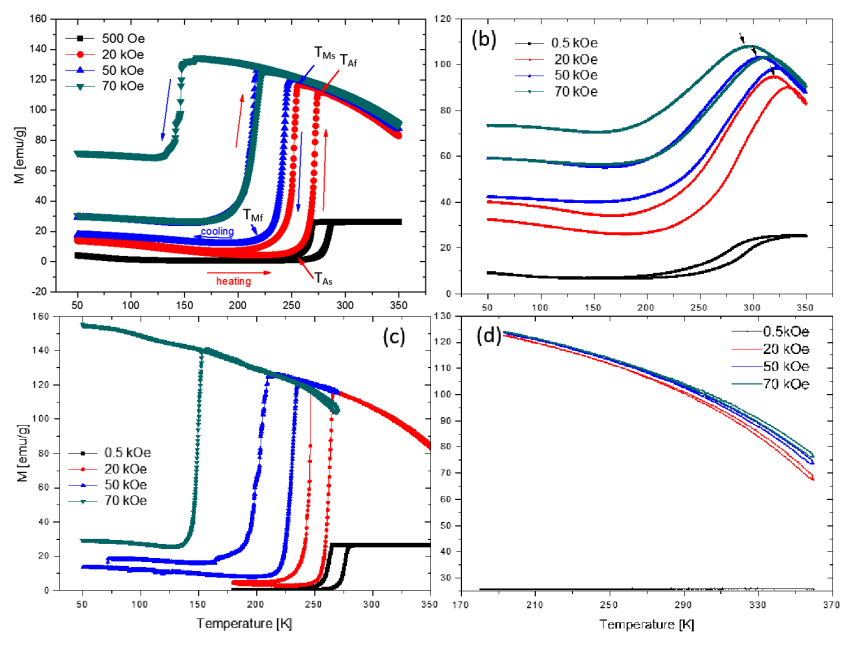

Fig. 3. Magnetization versus temperature curves of the B000 (a), B005 (b), B010 (c), B100 (d).

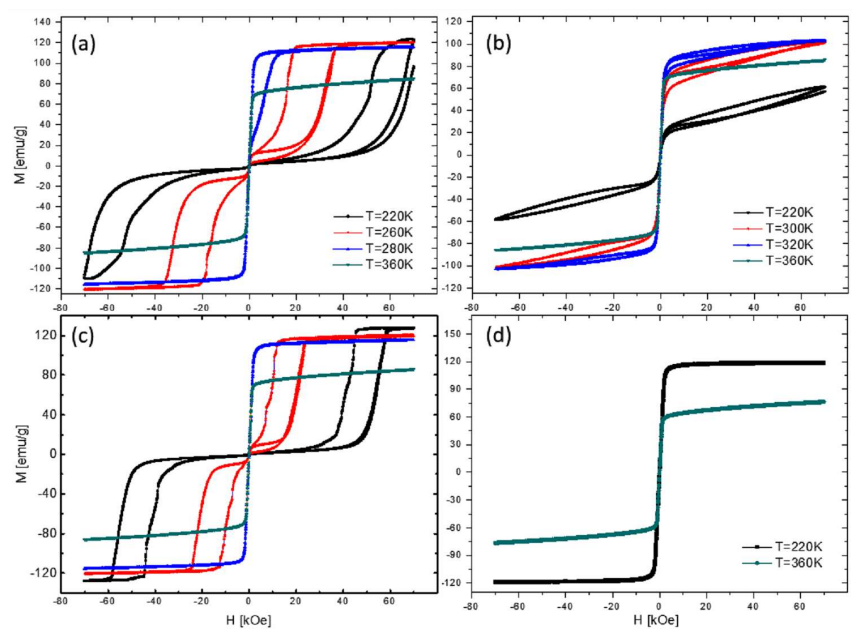

Fig. 4. Magnetization versus magnetic field curves of the B000 (a), B005 (b), B010 (c), B100 (d).

The curves at $220 \mathrm{~K}$ and $360 \mathrm{~K}$ exhibit a typical ferromagnetic behavior (Fig. 4a), whereas curves taken at
$260 \mathrm{~K}$ and $280 \mathrm{~K}$ show large hysteresis as a function of magnetic field. This behavior is characteristic for metamagnetic materials and is connected with the magnetic induced reverse transformation. Results obtained from magnetization vs. magnetic field curves are in good agreement with this depicted in Fig. 3. For specimen with the 0.05 at. $\%$ of $\mathrm{B}$ addition one can see the small narrow loops at 220,300 , and $320 \mathrm{~K}$ in the magnetization curves. In this plot (even better than in the thermomagnetization ones) can be seen that magnetically induced transformation occurs only in small part of the matrix. For the specimens B050 and B100 (where transformation was not observed) magnetization curves were recorded only at $200 \mathrm{~K}$ and $360 \mathrm{~K}$.

\section{Summary}

Series of the $\mathrm{Ni}_{45.5-x} \mathrm{Co}_{4.5} \mathrm{Mn}_{36.6} \operatorname{In}_{13.4} \mathrm{~B}_{x}$ [at.\%] ( $x=$ $0,0.05,0.1,0.5,1$ ) (denoted respectively as B000, B005, $\mathrm{B} 010, \mathrm{~B} 050, \mathrm{~B} 100)$ alloys were examined in terms of the structure and magnetic properties. Depending on the boron addition studied alloys show different microstructures at the room temperatrure: single phase (parent for B000) or two phase (parent+martensite for B005 or parent+precipitates for B010-B100). In the studied alloys the $L 2_{1}$ parent phase (B000-B100), $7 \mathrm{M}$ monoclinic martensite (B005) and $\mathrm{M}_{23} \mathrm{~B}_{6}(\mathrm{~B} 010-\mathrm{B} 100)$ of precipitates crystal structures were determined by TEM. Boron addition promotes precipitation of the second phase whose volume fraction increases with the boron addition. Additionally, boron influences martensitic transformation temperatures as well as magnetic properties. For alloys consisting $0.5-1.0$ at.\%B the transformation is hindered. Martensitic transformation temperature depends mainly on the electron to atom ratio (e/a) which is a function of the chemical composition. For the alloys consisting of Co-rich precipitates, e/a ratio of the matrix decreases. As a result the transformation temperatures decrease as well (B010). The higher volume fraction of the precipitates the lover martensitic transformation temperatures. Additionally, precipitates create kind of "barriers" which may even suppress the thermally activated martensitic transformation (B50-B100). In B000, B005, and B010 alloys magnetic field-induced reverse transformation (metamagnetic transformation) was observed. The decrease of the martensite characteristic temperatures with the magnetic field for B000 and B010 is over $120 \mathrm{~K}$ whereas for B005, in which only some part of the matrix transforms magnetically, is only $25 \mathrm{~K}$. To sum up, magnetic and structural properties of the $\mathrm{Ni}-$ Co-Mn-In metamagnetic shape memory alloys can be tuned by combination of the chemical composition (boron addition), applied magnetic field and temperature.

\section{Acknowledgments}

Research was financially supported by the Polish Ministry of Science and Higher Education under the project no. N507 518639. 


\section{References}

[1] K. Ulakko, J. Mater. Eng. Perform. 5, 405 (1996).

[2] A. Sozinov, A.A. Likhachev, N. Lanska, K. Ullakko, Appl. Phys. Lett. 80, 1746 (2002).

[3] E. Stern-Taulats, P.O. Castillo-Villa, L. Mañosa, C. Frontera, S. Pramanick, S. Majumdar, A. Planes, J. Appl. Phys. 115, 173907 (2014).

[4] R.Y. Umetsu, X. Xu, R. Kainuma, Scr. Mater. 116, 1 (2016).

[5] R. Kainuma, Y. Imano, W. Ito, Y. Sutou, Nature 439, 957 (2006).

[6] H. Morawiec, T. Goryczka, A. Drdzeń, J. Lelątko, K. Prusik, Solid State Phenom. 154, 133 (2009).
[7] K. Prusik, K. Bałdys, D. Stróż, T. Goryczka, J. Lelątko, Solid State Phenom. 203-204, 306 (2013).

[8] S. Tamirisakandala, R.B. Bhat, J.S. Tiley, D.B. Miracle, Scr. Mater. 53, 1421 (2005).

[9] M. Ramudu, A. Satish Kumar, V. Seshubai, Intermetallics 28, 51 (2012).

[10] S.N. Ghali, H.S. El-Faramawy, M.M. Eissa, J. Miner. Mater. Character. Eng. 11, 995 (2012).

[11] K. Prusik, E. Matyja, M. Zubko, M. Kubisztal, A. Chrobak, Acta Phys. Pol. A 130, 1023 (2016).

[12] Y. Sutou, R. Kainuma, K. Ishida, M. Taya, Proc. SPIE 5053, 159 (2003). 